\title{
Le boom de l'anacarde en Côte d'Ivoire: transition écologique et sociale des systèmes à base de coton et de cacao
}

\author{
François Ruf ${ }^{1,2, *}$, Siaka Kone ${ }^{3}$ et Boniface Bebo ${ }^{4}$ \\ ${ }^{1}$ CIRAD, UMR ART-DEV, Abidjan, Côte d'Ivoire \\ 2 ART-DEV, CIRAD, Univ. Montpellier, Montpellier, France \\ ${ }^{3}$ École supérieure d'agronomie (ESA), Institut national polytechnique Félix Houphouët Boigny (INPHB), BP 1313, Yamoussoukro, Côte \\ d'Ivoire \\ ${ }^{4}$ Structure agricole de développement rural de Côte d'Ivoire (SADRCI), Yamoussoukro, Côte d'Ivoire
}

\begin{abstract}
Résumé - Le dynamisme agricole de la Côte d'Ivoire continue de surprendre les marchés de matières premières, en particulier ceux du cacao et du caoutchouc. Le pays s'empare aussi de la place de premier producteur mondial d'anacarde. Comment interpréter ce boom? Il est en partie lié aux marchés (stagnation des prix du coton et du cacao, hausse du prix de l'anacarde sur deux périodes), mais les déterminants sont beaucoup plus complexes. L'hypothèse de l'étude est que l'adoption de l'anacardier relève en partie d'une transition écologique d'adaptation à l'épuisement de la rente forêt et au changement climatique, au coût croissant des intrants chimiques pour le coton et le cacao, mais répond aussi à l'insécurité foncière. Cette hypothèse a été testée en 2016-2017 sur 6 sites d'étude, choisis selon un gradient nord-sud: Mankono, Konanhiri, Bonon, Yamoussoukro, Bayota-Gagnoa et Soubré, à raison de 40 à 100 exploitations par site, avec des actualisations partielles en 2018. Ces enquêtes confirment l'hypothèse. L'anacardier, arbre robuste, résistant aux sécheresses, évite pour l'instant aux planteurs tout recours aux intrants chimiques et au crédit, reconstitue un ombrage forestier et est aussi un marqueur de terre (il apporte une sécurité foncière informelle dans le village d'origine des planteurs migrants, mais aussi dans leur village cacaoyer). Il devient l'outil d'une transition écologique et sociale. Cette transition inclut des flux significatifs d'information, de travail et d'investissement entre les deux espaces économiques. Elle relève essentiellement d'une innovation paysanne. En zone cacaoyère, l'anacardier, dans sa dimension agroforestière sous forme d'association avec le cacaoyer, réduit la mortalité des jeunes plants de cacao, et son adoption prend tout son sens de transition agroécologique. L'agriculture familiale est-elle en train de réaliser seule ce que l'industrie du chocolat promet sur le papier: une cacaoculture «zéro-déforestation» et durable?
\end{abstract}

Mots clés : changement climatique / swollen shoot / diversification / déforestation / agroforesterie / insécurité foncière

Abstract - Côte d'Ivoire's cashew nut boom: A social and ecological transition of the cotton and cocoa systems. Côte d'Ivoire's agricultural dynamism continues to surprise commodity markets, especially those of cocoa and rubber. The country has also become the world's leading producer of cashew nuts. How to understand this boom? It is partly driven by markets (stagnation of cotton and cocoa prices versus rising prices of cashew nuts over two periods) but its determining factors are much more complex. The global hypothesis of this study is that the adoption of cashew tree is an adaptation to the loss of forest rent and to climate change, to the increasing cost of chemical inputs for cotton and cocoa, but also responds to land insecurity. An in-depth study was conducted in 2016-2017 in 6 sites chosen along a north-south gradient: Mankono, Konahiri, Bonon, Yamoussoukro, Bayota-Gagnoa and Soubré, with 40 to 100 farms per site, with partial updates in 2018. The survey confirms the hypothesis. Cashew tree, a robust, droughttolerant tree, for the time being avoids planters relying on chemical inputs and credit, rebuilds a kind of forest canopy, but is also a land marker (it brings an informal land security in the home villages of the migrants but also in their cocoa villages). It becomes the tool of a social and ecological transition. That transition includes significant information, labour and investment flows between the two economic spaces. It is essentially a smallholders' innovation. In the cocoa regions, in its agroforestry version made of cocoa and

\footnotetext{
$\overline{\text { *Auteur de correspondance }}:$ francois.ruf@cirad.fr
} 
cashew intercropping, the cashew tree reduces the mortality of cocoa seedlings, and its adoption takes its full dimension of agro-ecological transition. Is family farming alone achieving what the chocolate industry promises on paper: a "zero-deforestation" and sustainable cocoa farming?

Keywords: climate change / swollen shoot / diversification / deforestation / agroforestry / food insecurity

\section{Introduction}

Le dynamisme agricole de la Côte d'Ivoire continue de surprendre les marchés de matières premières. En dépit de la baisse structurelle du prix du cacao, des années de troubles politico-militaires (2002-2011) et des prévisions alarmistes de l'industrie évoquant une pénurie de chocolat, le pays renforce son rang de premier producteur mondial de cacao en franchissant le seuil de 2000000 tonnes de cacao. Parallèlement, la grande région ivoirienne de production de cacao - de plus en plus «post-forestière» du fait de la déforestation massive dans le tiers sud du pays - poursuit le développement accéléré de l'hévéaculture: la production de caoutchouc est passée de 60000 tonnes en 1993 à 800000 tonnes en 2018. En zone de savane, la production de coton, plus chaotique, a repassé le seuil des 400000 tonnes/an de coton graine à partir de 2017-2018.

Mais le changement le plus important concerne la production de la noix de cajou ou anacarde. En deux décennies, le pays s'est emparé presque discrètement de la place de premier producteur mondial. De 8500 tonnes en 1989 , la production ivoirienne d'anacarde a dépassé 70000 tonnes en 1999, puis a bondi à 350000 tonnes en 2010 (Dugué, 2002; Kone, 2014 ; Banque Mondiale, 2015). Malgré la sécheresse de 2016 et les ventes clandestines vers le Ghana, le seuil de 700000 tonnes a été officiellement franchi en 2017.

Dans un premier temps, le boom de l'anacarde s'est produit principalement en zone de savane (Dugué, 2002; Diomandé, 2002; Kone, 2014). Mais la noix de cajou s'est également invitée vers le sud dès les années 2000, dans les zones cacaoyères de contact forêt-savane telles que celles de Tanda, mais également dans les anciennes boucles du cacao vers M'Bahiakro à l'Est et Bouaflé à l'Ouest, et plus récemment, vers 2010, au cœur de régions cacaoyères comme à Bayota, au nord de Gagnoa (Ruf, 2018). Les deux processus de progression de la production d'anacarde en savane et d'émergence plus récente dans le sud sont partiellement liés. De nombreux planteurs d'anacardiers dans le nord sont des planteurs de cacaoyers dans le sud, ou du moins parents. Par exemple, les planteurs de cacaoyers à San Pedro (sud-ouest du pays), ayant migré dans les années 1980 depuis Bondoukou et Tanda (nord-est), envoient leurs épouses planter des anacardiers au village d'origine (Ruf, 2016).

L'anacarde apparaît donc comme une culture de diversification dans deux espaces économiques reliés par des réseaux familiaux : zones dites de savane mais reconstituant une forme de couvert forestier avec l'anacardier, et zones dites forestières, mais de plus en plus privées de leur rente forêt. Indépendamment de l'anacarde, l'enjeu économique global de la zone cacaoyère est de surmonter la disparition de la rente forêt dans un milieu devenu «post-forestier» (Ruf, 1995; Léonard et Oswald, 1996; Sanial, 2018). Comment analyser cette double diversification dans les zones cacaoyères et cotonnières, basée sur l'anacardier et évoluant en boom économique national?

Une synthèse sur la diversification des cultures pérennes tropicales (Ruf et Schroth, 2013) conclut à cinq déterminants majeurs de cette diversification, presque toujours présents dans les évolutions des économies de plantation:

- le marché constitue un déterminant majeur de l'intérêt des villageois à entamer la diversification. Par exemple, pour les planteurs de cacao, le mouvement vers l'hévéa répond en partie à la hausse des cours du caoutchouc à la fin des années 2000 et à la quasi-mensualisation des revenus (Losch, 1990; Fiko et Yao, 2013; Oura Kouadio, 2017);

- les initiatives de l'industrie en aval du produit et les projets de développement soutenus par des bailleurs de fonds stimulent la diversification par la demande ;

- les politiques publiques peuvent aussi stimuler la diversification par le biais de taxations différentielles des diverses cultures pérennes ;

- mais le premier déterminant structurel de la diversification serait l'ensemble des changements biologiques, écologiques et climatiques opérant au cours du déroulement d'un cycle fondé sur une quasi-monoculture. Par exemple, l'adoption de l'hévéa en Côte d'Ivoire et au Ghana répond en grande partie au problème du vieillissement des plantations de cacao et à la dégradation des ressources naturelles (sol, végétation spontanée). Cette dégradation et le changement climatique accroissent les difficultés de replantation cacaoyère, alors que la nouvelle culture, l'hévéa, valorise des sols en voie d'acidification sous jachère et échappe un temps aux maladies. L'hévéa génère ainsi une nouvelle rente « hévéa et post-forestière » se substituant à la rente « forêt » réalisée et consommée par le cacao;

- enfin, des facteurs sociaux participent au processus de diversification après le déroulement d'un cycle de cacao. $\mathrm{Au}$ fil des décennies, la pression foncière augmente, générant des conflits et des stratégies pour occuper les espaces encore «libres» avec des arbres plantés, marqueurs de terres. Ainsi, les fils et petit-fils d'immigrants plantent des hévéas à la hâte pour défendre leurs terres contre les velléités de reprise par la seconde génération d'autochtones.

Quels sont donc les facteurs explicatifs du boom de l'anacardier en Côte d'Ivoire? Est-il lié aux politiques publiques favorisant cette nouvelle culture ou indirectement à celles des «anciennes» cultures que sont le cacao et le coton? Le développement de la production d'anacarde relèvet-il plutôt d'une simple adaptation au marché (d'une incitation par les prix relatifs entre ancienne et nouvelle culture) ou au contraire du changement écologique et climatique en lien avec la raréfaction des bonnes terres? La diffusion massive de l'anacardier répond-elle à un besoin de réduction des intrants chimiques en agriculture et en particulier sur le cacaoyer et le cotonnier? Enfin, l'adoption de l'anacardier sert-elle à affirmer 


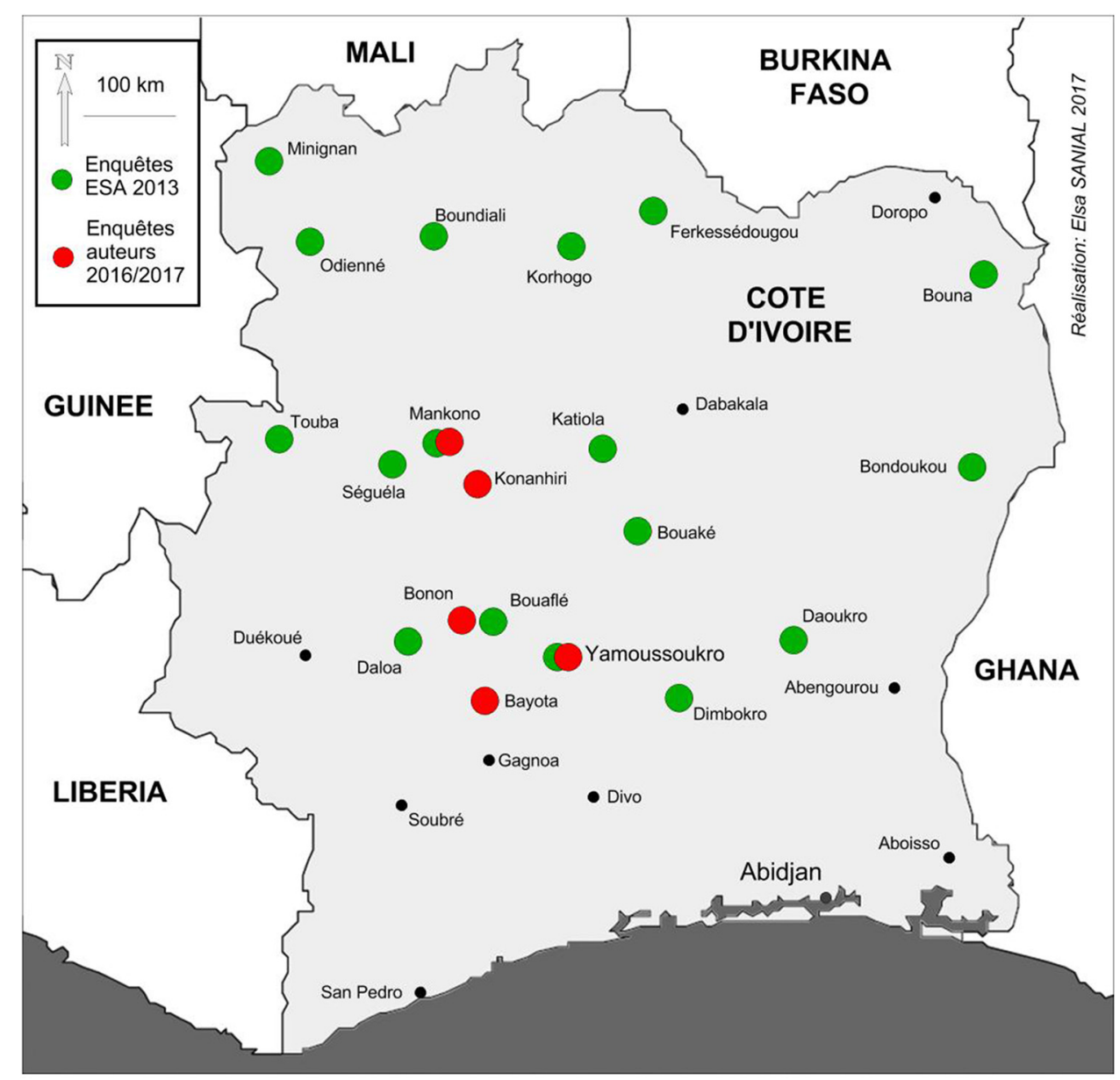

Fig. 1. Localisation des sites d'étude.

Fig. 1. Location of survey sites.

un droit de propriété sur la terre? Ce boom de l'anacarde correspond-il à une double transition écologique et sociale?

\section{Méthodes}

Nous avons d'abord analysé les statistiques nationales de production et de prix disponibles, pour l'anacarde, le coton et le cacao. Ensuite, les déterminants supposés de ce boom ont été approfondis sur 5 sites d'études en 2017. Nous sommes partis de la carte des sites d'anacardiers identifiés au plan national par Kone (2014), puis nous en avons choisi 4 selon un gradient nord-sud (Fig. 1) : Mankono pour la zone de savane, Konanhiri pour le nord de la zone de contact forêt-savane, les environs de Bouaflé (Bonon et parc national de la Marahoué) et les environs de Yamoussoukro. Un $5^{\mathrm{e}}$ site plus au sud, BayotaGagnoa (village de Konankouassikro), au cœur de la zone cacaoyère, en plus d'être soumis au questionnaire « anacarde » comme tous les sites, avait auparavant été suivi à 15 ans d'intervalle. C'est ce qui nous avait permis de repérer l'adoption de l'anacarde en 2017 dans une zone dite forestière où il n'était pas attendu. Ces 5 sites ont été choisis pour l'émergence de l'anacardier dans leurs systèmes agraires et pour ce gradient géographique montrant que l'anacardier n'est plus seulement un arbre de savane. En complément ont été mobilisées des données également collectées à 15 ans d'intervalle dans un $6^{\mathrm{e}}$ village plus au sud, dans la boucle du cacao de Soubré, où l'anacardier est pour l'instant cantonné à quelques essais par les migrants (mais qui peuvent avoir des plantations dans leur village d'origine en savane. Dans chacun des 6 sites, entre 40 et 100 planteurs ont été interrogés au hasard dans les villages (Fig. 1).

Au-delà des données chiffrées, les histoires locales de chacun de ces 6 sites et d'autres villages, par exemple les alentours de Daloa, Issia et Vavoua, ont également été exploitées pour améliorer l'analyse de nos résultats.

\section{Le boom de l'anacarde à l'échelle nationale: premiers éclairages locaux}

Le boom de l'anacarde contraste avec la stagnation de la production de coton (Fig. 2). Alors que les prix du coton sont restés faibles sur une longue période, ceux de l'anacarde ont été marqués par deux phases de prix élevés (1994-2000 et 2014-2017, jusqu'au tout début de 2018), qui ont joué un rôle déclencheur (Fig. 3).

L'anacardier avait été promu dans les années 1950 par les services de l'agriculture pour ses services écologiques: il résiste aux sécheresses. Jusque dans les années 1990, il intéressait peu les agriculteurs. Mais quand les acheteurs asiatiques d'anacarde sont venus prospecter dans le nord de la 


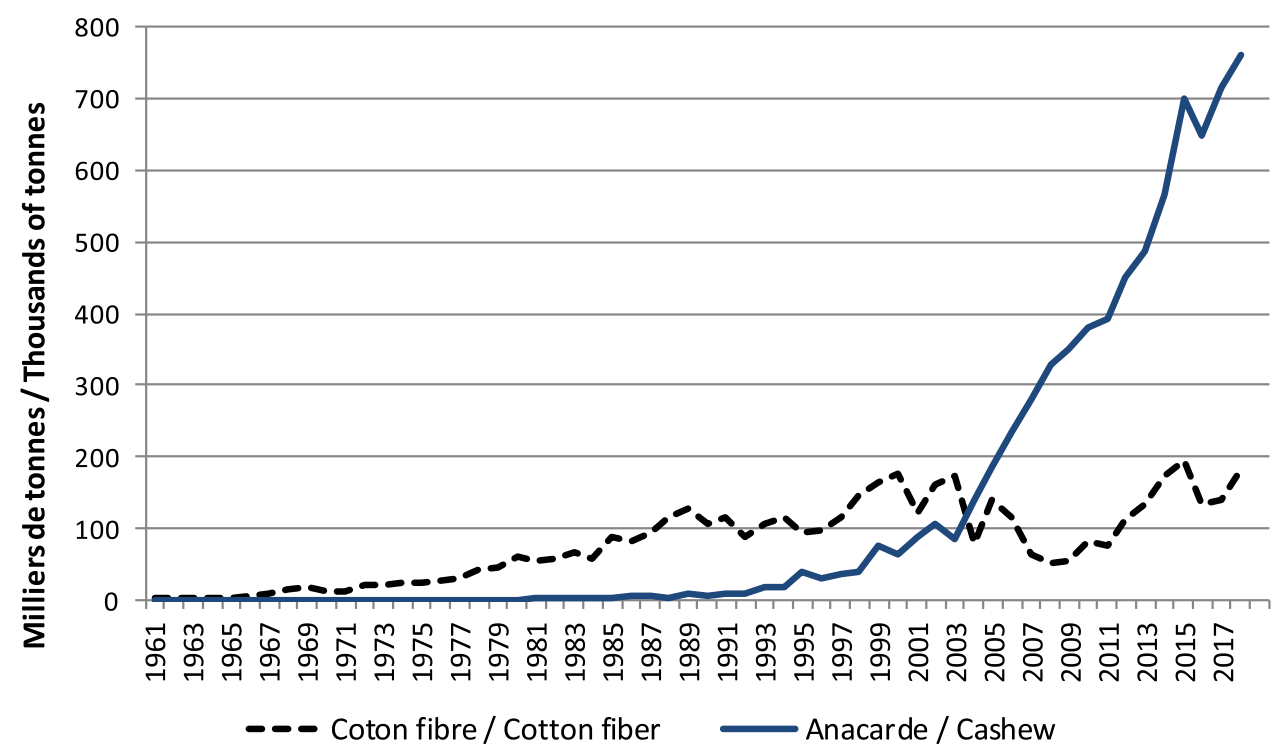

Fig. 2. Production d'anacarde et de coton-fibre en Côte d'Ivoire de 1961 à 2017. Source: FAOSTAT et divers sites web après 2014. Fig. 2. Production of cashew and cotton-fiber in Côte d'Ivoire from 1961 to 2017. Source: FAOSTAT and various web sites after 2014.

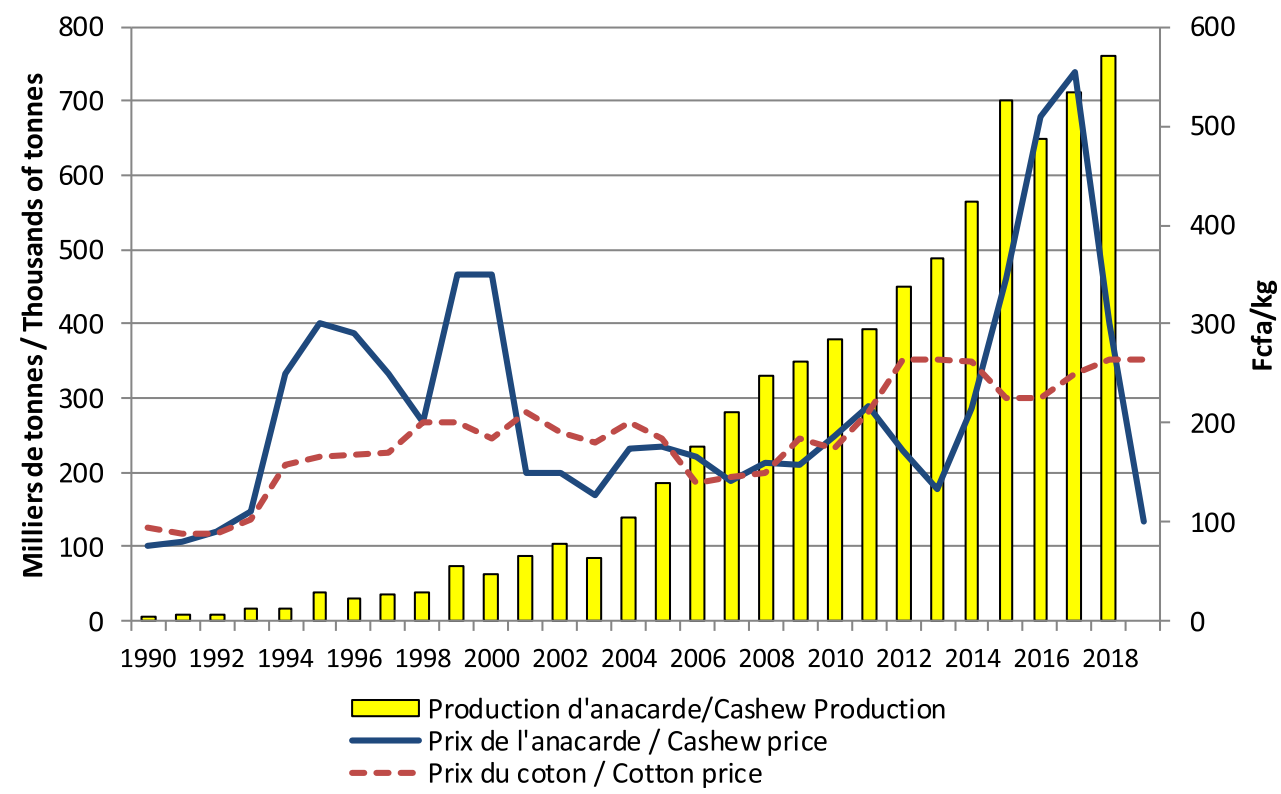

Fig. 3. Production nationale d'anacarde, prix courants de l'anacarde et du coton-graine payés aux producteurs de 1990 à 2018 . Source: FAOSTAT, Intercoton, Rongead 2010, 2014, observations auteurs 2017-2019.

Fig. 3. National production of cashew, nominal prices of cashew and cotton paid to producers from 1990 to 2018. Source: FAOSTAT, Intercoton, Rongead 2010, 2014, observations auteurs 2017-2019.

Côte d'Ivoire à partir des années 1990-2000, les agriculteurs ont valorisé cet arbre (Basset, 2009). Ainsi, des plantations d'anacardiers se sont créées massivement entre 2001 et 2013, une période prolongée de stagnation du prix du coton graine. Mais le marché et les prix relatifs du coton et de l'anacarde ne suffisent pas à expliquer l'ampleur du boom de l'anacarde. D'autres facteurs sont intervenus.

L'un d'entre eux est la baisse structurelle des revenus du cacao, résultant de la baisse des prix (Fig. 4), puis de celle des rendements sous l'effet du vieillissement et des maladies des cacaoyers, dans bien des régions. Un des cas d'école de cet effet de cycle est celui de Bonon et du Parc de la Marahoué, où les rendements pouvaient approcher 2 tonnes/ha dans les années 1980 (ENGREF, 2002), alors que les plantations ont dépéri, puis sont mortes dans les années 2010 sous l'impact du swollen shoot et de la sécheresse. Bonon est un cas extrême, mais dans plusieurs régions de la zone cacaoyère, l'érosion des revenus du cacao a d'abord généré des frustrations et des retours de migrants dans leur village d'origine pour développer l'anacarde, et donc initier une diversification des cultures, d'abord en zone cotonnière, mais aussi en zone cacaoyère. C'est le cas de Konanhiri. Dans leurs villages d'émigration 
cacaoyère, les planteurs originaires de Konanhiri ont subi le vieillissement des cacaoyers, "l'usure» du milieu, la baisse des rendements et des revenus. L'insécurité foncière a fragilisé la situation des immigrants. Les deux phénomènes les ont incités à investir dans leur village d'origine (Encadré 1). La course à la terre s'est alors engagée au village d'origine et l'insécurité foncière est également devenue un moteur de plantation d'anacardiers en savane (Ruf, 2016).

Encadré 1. Kounanhiri : du coton à l'anacarde en passant par le cacao.

\section{0-1970. $1^{\mathrm{e}}$ génération : le coton}

Dans les années 1950-1970, les populations de Kounanhiri avaient adopté le coton comme culture de rente, peu rentable. À la fin de la récolte, les paysans devaient encore de l'argent à la Compagnie ivoirienne pour le développement des textiles (CIDT) qui fournissait les intrants aux producteurs en début de culture, et pouvaient rester endettés 2 ou 3 ans. Cette intégration verticale décourageait la jeune génération, laquelle est partie «à l'aventure » en zone forestière.

1970-1990. $2^{\mathrm{e}}$ génération : abandon du coton, migration et cycle « cacao »

Les premiers planteurs Ouan se sont installés dans les zones forestières pour défricher, planter des caféiers, puis des cacaoyers. Le coton et son espace économique ont ainsi été abandonnés pendant près de $20 \mathrm{ans}$ par les populations Ouan de Kounanhiri au profit de nouveaux espaces économiques basés sur le cacao. Mais lorsque les prix du cacao et du café ont chuté dans les années 19881993, suivis des premières baisses de rendement par vieillissement des cacaoyères, du manque de terres pour les cultures vivrières, cette génération s'est vue confrontée au même problème d'effondrement de revenus que leurs parents avec le coton. Par ailleurs, au fil des années et notamment avec l'avènement du multipartisme en Côte d'Ivoire, les autochtones de l'ouest ont commencé à revendiquer leurs droits sur «la terre de leurs ancêtres ». Certains ont alors eu l'idée de retourner au village d'origine, mais cette fois pas pour le coton.

1991-2010. $2^{\mathrm{e}}$ et $3^{\mathrm{e}}$ génération : retour au village et adoption de l'anacarde

L'objectif était d'abord d'assurer la production d'igname. Puis, une nouvelle culture, l'anacardier, allait être valorisée par ces planteurs qui étaient retournés au village d'origine. Le processus a pris plusieurs années. Les migrants Ouan, devenus vieux, ont commencé à revenir au village d'origine, avec certains de leurs fils, pour créer une plantation d'anacarde.

2011-2015. $3^{\mathrm{e}}$ et $4^{\mathrm{e}}$ génération : cacao au nord, anacarde au sud

Une inversion des innovations et des flux de migration s'est produite, avec une partie des jeunes plantant le cacao au village d'origine au nord, dans les quelques galeries forestières parsemant le terroir de savane, suivis de nouveaux immigrants, tandis qu'ils allaient essayer l'anacarde au sud dans le village cacaoyer du réseau familial.

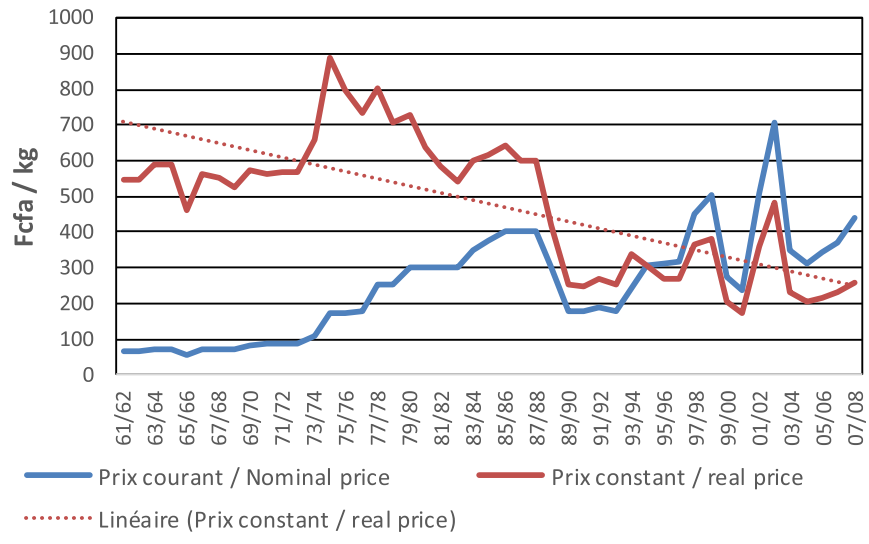

Fig. 4. Prix courants et constants du cacao payés aux producteurs de 1961 à 2008. Source: caisse de stabilisation jusqu'en 1987, puis relevés auteurs.

Fig. 4. Nominal and real prices of cocoa paid to producers from 1961 to 2008. Source: Ivorian cocoa marketing board until 1987, then authors' records.

Ces histoires locales invitent donc à suivre la piste de multiples transitions sociales et agroécologiques dans les deux espaces économiques, à la fois au village d'origine et au village de migration cacaoyère. Mais explorons d'abord la composante économique, celle de la dépendance aux intrants dans le binôme de savane «coton-anacarde» et dans le nouveau binôme «cacao-anacarde» en zone post-forestière. Nous allons vérifier que ce «nouvel arbre», l'anacardier, a bien la fonction de libérer les agriculteurs de la dépendance aux intrants, initiant ainsi une transition agroécologique.

\section{Dépendance et indépendance vis-à-vis des intrants chimiques}

L'anacardier, arbre robuste, résistant à la sécheresse et aux feux (système racinaire résistant, canopée couvrant le sol et éliminant les herbes), est cultivé sans aucun intrant chimique, d'ailleurs peu ou pas conseillé dans les manuels (Ouattara et al., 2012). C'est la grande différence entre l'anacardier et la culture cotonnière depuis des décennies. Pour le cacao, la dépendance aux produits chimiques est plus récente, mais s'accélère.

\subsection{Une culture cacaoyère post-forestière progressant vers «le chimique»}

Dans les années 1970-1980, en pleine conquête pionnière aux dépens des forêts du pays, à peine $25 \%$ des planteurs de cacao utilisaient des pesticides, aucun n'utilisait d'engrais chimique ou organique ni d'herbicides (Ruf, 1995). Grâce à la rente forêt, la majorité des planteurs produisait alors du cacao «biologique » par défaut, sans le savoir.

À la fin des années 1990, l'utilisation des pesticides sur cacaoyers s'est accrue et les planteurs ont découvert les engrais minéraux. Les herbicides se sont diffusés massivement dans les années 2000 (Ruf, 1999, 2018).

Dans les années 2010, la zone cacaoyère a dû composer avec des sols dégradés, une pression accrue des bio-agresseurs et des mauvaises herbes, un climat plus sec et plus aléatoire. La 
Tableau 1. Proportion de planteurs utilisant des intrants dans les exploitations cacaoyères à Konankouassikro (région de Bayota, au nord de Gagnoa) en 1998 et 2016.

Table 1. Proportion of farmers using inputs on cocoa farms in Konankouassikro (Bayota region, north of Gagnoa) in 1998 and 2016.

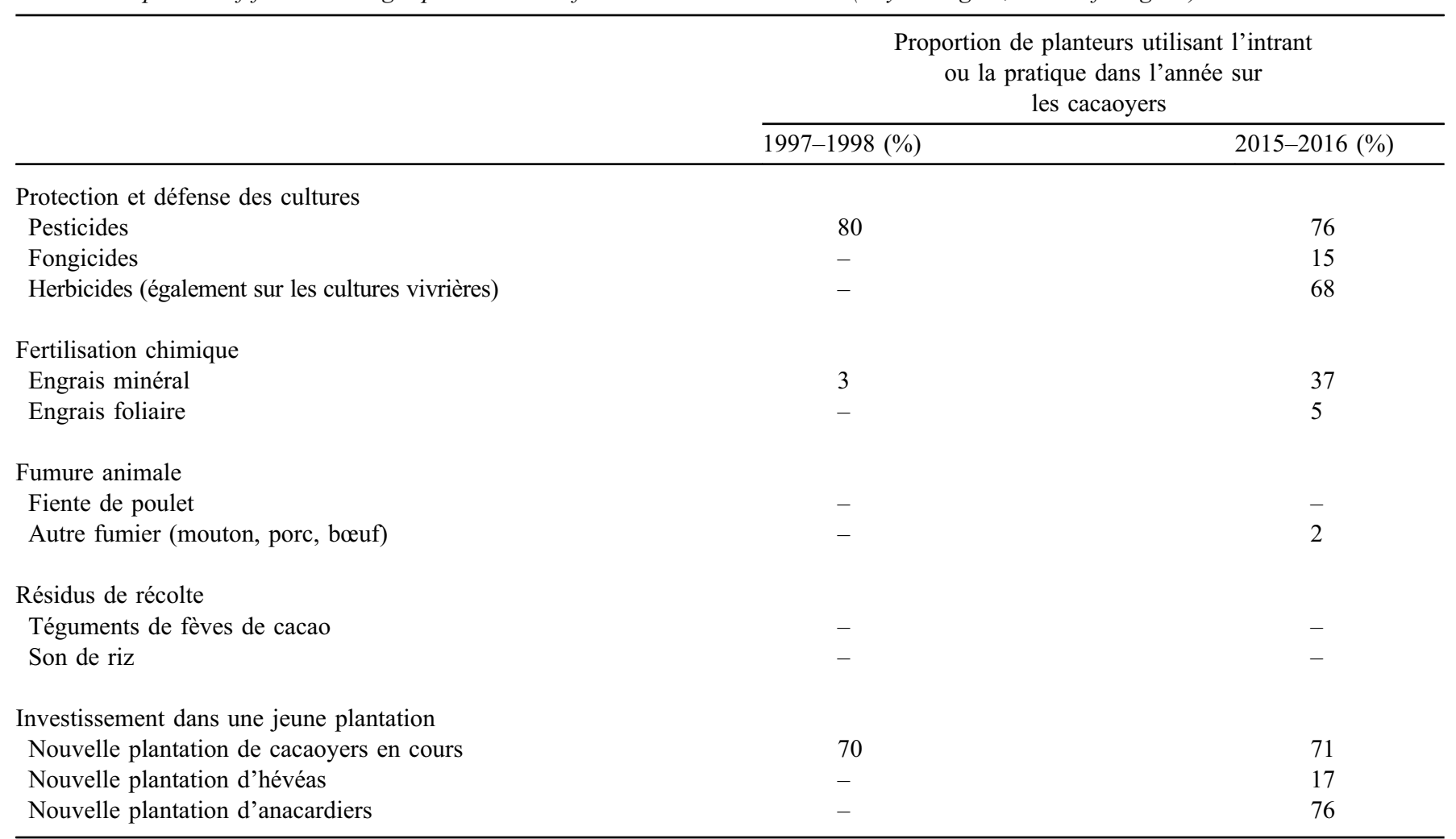

Source: enquêtes auteurs, 66 exploitations en 1998, 56 en 2016.

rente forêt a été consommée et les planteurs ont tenté de compenser sa perte par de nouveaux intrants. Dans le site de Bayota, représentant ici les zones cacaoyères vieillissantes, la consommation de pesticides concernait déjà $80 \%$ des planteurs à la fin des années 1990 et est restée à ce niveau dans les années 2010 (Tab. 1). Les engrais, quasiment inconnus en 1997-1998, sont passés à un taux d'adoption de 30 à $40 \%$ en 2016. Les herbicides, totalement inconnus en 1997, permettant de réduire le travail de désherbage, concernent maintenant près de $70 \%$ des planteurs (en priorité pour leurs champs de riz de bas-fond qui se développent précisément grâce à l'herbicide, mais aussi pour le cacao). Dans la boucle du cacao de Soubré et San Pedro, les planteurs villageois ont introduit de la fiente de volaille importée des élevages de l'Est du pays pour assurer la réussite de leurs nouvelles cacaoyères sur sol dégradé, puis progressivement du fumier de mouton et de porcs élevés localement dans les exploitations cacaoyères. Enfin, les fertilisants organiques à base de résidus de récolte (son de riz, téguments de fèves de cacao) ont également émergé (Tab. 2).

Ainsi, après 35 ans de déforestation pour planter des cacaoyers et de valorisation des ressources naturelles et de « la rente forêt», les pratiques agricoles en zone forestière ivoirienne se sont modifiées et le recours aux intrants chimiques s'est développé. Mais de ce fait, le coût d'entretien des plantations adultes a augmenté. En 2017, hors travail d'application, ce coût s'élevait à environ 200 euros; par tonne de cacao produite et payée moins de 1100 euros; aux planteurs.
Cette augmentation des coûts, dans un contexte de baisse de prix du cacao, a contribué à déclencher des innovations villageoises. L'association anacardier-cacaoyer est devenue un nouveau cas d'école. Les planteurs qui étaient retournés dans leur village d'origine pour investir dans cet arbre sont revenus avec leurs semences. Ils étaient conscients que cet arbre, résistant à la sécheresse et pour l'instant aux maladies, n'avait pas besoin d'intrants, même sur des sols appauvris, tant en phase de création de la plantation qu'en phase productive (Dugué, 2002). L'installation d'une plantation d'anacardiers était très peu coûteuse car elle se faisait par semis de semences «tout venant» dans des parcelles en production vivrière pendant deux à trois années. L'entretien de ces cultures alimentaires assurait aussi le bon développement des jeunes anacardiers qui rentraient en production en année 3 ou 4 (Dugué, 2002). L'anacardier présentait ainsi des propriétés que le cacaoyer avait perdues avec le changement écologique.

Cette adoption de l'anacardier marque bien le début d'une transition agroécologique, réduisant les coûts et les risques liés aux intrants chimiques.

\subsection{Un coton « chimique » de longue date}

Pour obtenir un rendement économiquement acceptable, le cotonnier nécessite d'utiliser des engrais minéraux et des insecticides (Cretenet et Vaissayre, 1986 ; Cretenet, 1987 ; Fok et al., 2015). En 2012, les optima économiques se situaient 
Tableau 2. Proportion de planteurs utilisant des intrants dans les exploitations cacaoyères du Bas Sassandra (Soubré et San Pedro) en 1982 et 2016.

Table 2. Proportion of farmers using inputs on cocoa farms in low Sassandra (Soubré and San Pedro) in 1982 and 2016.

\begin{tabular}{|c|c|c|}
\hline & \multicolumn{2}{|c|}{$\begin{array}{l}\text { Proportion de planteurs utilisant l'intrant ou la pratique } \\
\text { dans l'année sur les cacaoyers }\end{array}$} \\
\hline & $1982-1983(\%)$ & 2015-2016 (\%) \\
\hline \multicolumn{3}{|l|}{ Protection et défense des cultures } \\
\hline Pesticides & 25 & 96 \\
\hline Fongicides & - & 15 \\
\hline \multicolumn{3}{|l|}{ Fertilisation chimique } \\
\hline Engrais minéral & - & 36 \\
\hline Engrais foliaire & - & 7 \\
\hline \multicolumn{3}{|l|}{ Fumure animale } \\
\hline Téguments de fèves de cacao & - & 6 \\
\hline Son de riz & - & 2 \\
\hline \multicolumn{3}{|l|}{ Investissement dans une jeune plantation } \\
\hline Nouvelle plantation de cacao en cours & 95 & 9 \\
\hline
\end{tabular}

Source: enquêtes auteurs, 150 exploitations en 1982, 121 en 2016.

théoriquement autour de 6 à 8 traitements insecticides par cycle cultural et $230 \mathrm{~kg} /$ ha d'engrais ; l'utilisation d'herbicides était également fréquente. L'application de ces préconisations était risquée et coûteuse. De 1977 à 1984, l'ensemble des intrants a été subventionné à $100 \%$ par l'État dans le cadre d'une politique de vulgarisation de masse. Ces subventions se sont arrêtées en 1984 (Cretenet et Vaissayre, 1986).

Dans les années 2010, on a constaté une grande variation des doses d'intrants chimiques par hectare, car les producteurs utilisaient ces intrants selon leurs moyens financiers (Fok et al., 2015). Néanmoins, même variées, les consommations restaient élevées. Selon Fok (2016), un quart à un tiers des exploitations cotonnières étaient toujours prêtes à investir fortement et à prendre des risques de non-remboursement du crédit de campagne qui leur était octroyé pour ces intrants. Mais la majorité d'entre eux ont réduit leur investissement en intrants $\mathrm{du}$ fait de l'augmentation des prix. Parallèlement à cette stratégie paysanne, les exploitations cotonnières ont diversifié en produisant de l'anacarde sans intrants, ce qui équivaut làaussi à une forme de transition écologique pour raisons économiques.

En 2014, $78 \%$ des exploitations cotonnières cultivaient aussi de l'anacardier (Fok et al., 2015). Mais nous avons observé des cas, en particulier dans la région de Mankono en 2017 , où l'essor de l'anacardier ne correspond pas seulement à la réduction des intrants chimiques appliqués au coton, mais s'est fait aussi au détriment des surfaces de cette culture annuelle, et au détriment des cultures vivrières comme constaté par la coopération allemande (projet PROFIAB) dans le nordest à Dabakala.

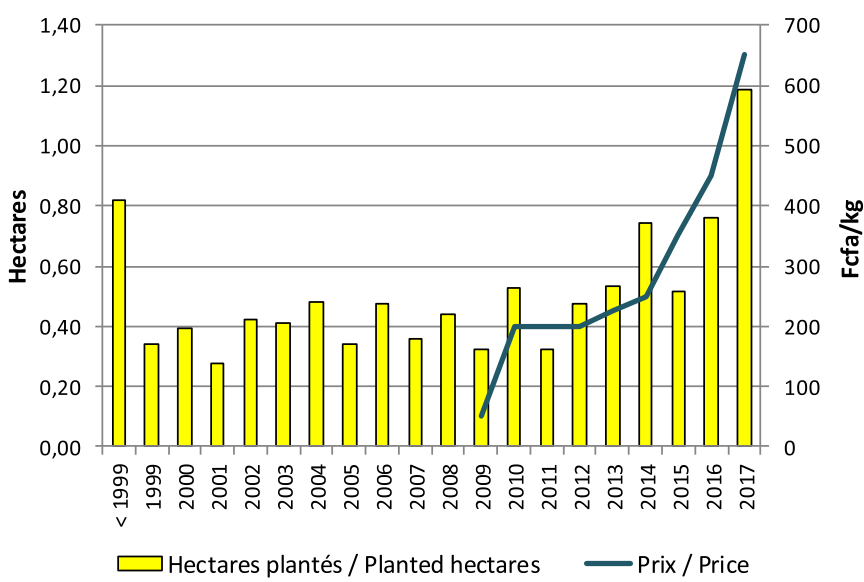

Fig. 5. Surface moyenne plantée annuellement par exploitation et prix courant de l'anacarde dans la région de Mankono. Source: déclarations des planteurs, enquêtes auteurs 2017.

Fig. 5. Average area planted annually by farm and nominal price of cashew nuts in the Mankono region. Source: farmers 'statements, data collected by authors in 2017.

\section{Des difficultés du coton au boom de l'anacarde: le cas de Mankono}

Dans la zone de Mankono, la culture de l'anacarde a été adoptée dans les années 1980, en petites superficies de 0,25 à 0,5 ha, côtoyant le coton, qui était alors la culture de rente dominante. À partir de 2013-2014, la hausse du prix courant 
Tableau 3. Contraintes et risques de la culture du coton cités par les planteurs, par opposition aux facilités de la culture de l'anacardier. Table 3. Constraints and risks of cotton cultivation cited by planters as opposed to the facilities of cashew cultivation.

\begin{tabular}{|c|c|c|}
\hline $\begin{array}{l}\text { Contraintes et risques cités par les } \\
\text { planteurs }\end{array}$ & Coton & Anacarde \\
\hline $\begin{array}{l}\text { 1. Besoin en capital et épargne pour } \\
\text { financer les intrants }\end{array}$ & $\begin{array}{l}\text { Élevé: «Le coton est une perte de temps pour } \\
\text { celui qui n'a pas les moyens" }\end{array}$ & Faible. Peu ou pas d'intrants \\
\hline $\begin{array}{l}\text { 2. Risque d'endettement. Soumission au } \\
\text { crédit }\end{array}$ & $\begin{array}{l}\text { Très élevé : «Nos parents faisaient le coton, ils } \\
\text { étaient pauvres et endettés » }\end{array}$ & $\begin{array}{l}\text { Pas de risque : "Il n'y a pas de crédit } \\
\text { dans l'anacarde» }\end{array}$ \\
\hline $\begin{array}{l}\text { 3. Surface et main-d'œuvre requise, } \\
\text { pénibilité du travail }\end{array}$ & $\begin{array}{l}\text { Élevées : "Pour avoir l'argent dans le coton, il } \\
\text { faut au moins 3-4 ha et des manæuvres, alors que } \\
\text { la main-d'œuvre manque }\end{array}$ & $\begin{array}{l}\text { Pas de contrainte. On peut cultiver } \\
\text { l'anacarde sans manœuvres. Le travail } \\
\text { est facile (notamment à la plantation, } \\
\text { dans les cultures vivrières) }\end{array}$ \\
\hline
\end{tabular}

de la noix de cajou et la stagnation du prix du coton ont entraîné le développement de l'anacarde (Fig. 5).

Parmi les 40 planteurs interrogés en 2017, à qui nous avons demandé la raison de l'adoption de l'anacarde, sans proposer de réponse prédéterminée, $50 \%$ citent spontanément la hausse récente du prix de l'anacarde, $30 \%$ évoquent un abandon du coton dans les 10 dernières années et $90 \%$ d'entre eux affirment qu'ils vont exclure le coton de leur exploitation, notamment les jeunes, pour trois grandes raisons dépassant le seul critère du prix (Tab. 3) :

- premièrement, les producteurs souhaitent être moins dépendants du crédit intrants et des difficultés structurelles de remboursement de ce crédit [«Avec le coton, on ne fait que payer des crédits. Il faut prendre son propre argent pour compléter le remboursement" (planteurs de Konahiri)], ce qui les amène à augmenter d'abord les surfaces en ignames précoces « déjà cultivées par nos parents » comme culture de rente, puis à adopter l'anacardier;

- deuxièmement, $30 \%$ des planteurs d'anacardiers en 2017 justifient leurs choix suite aux faibles performances économiques de leurs cultures cotonnières (faibles revenus et rémunération de la journée de travail) et à l'échec de leur implantation passée en ville comme transporteur, chauffeur, aide-chauffeur, gérant de moulin à céréales, maçon, couturier et «sans métier» à Abidjan... La création d'une plantation d'anacardiers, peu coûteuse si elle se fait sur une terre familiale, constitue un processus de réinsertion socialement reconnu dans le village. De plus, les premières ventes de noix de cajou arrivent en $3^{\mathrm{e}}$ ou $4^{\mathrm{e}}$ année après la plantation et l'existence de cette filière les incite à revenir dans leur village ;

- enfin, $10 \%$ des producteurs interrogés à Mankono font référence à un lien avec un village en zone cacaoyère. Une vieille cacaoyère, appartenant au producteur lui-même ou à un membre de sa famille, lui vient en soutien, notamment en fournissant de la main-d'œuvre pour la récolte des noix de cajou.

\section{Quand l'érosion cacaoyère et l'investissement dans l'anacarde inversent les flux de migration}

Historiquement, l'économie cacaoyère de la Côte d'Ivoire s'est construite à partir de migrations depuis le centre et le nord du pays et depuis le Burkina Faso vers le sud forestier. Au tournant des années 1990-2000 a débuté une inversion de flux, avec des migrations depuis les vieilles zones cacaoyères vers les nouvelles régions d'investissement dans l'anacarde et le cacao. $\mathrm{Au}$ sud, les villages comprenant des cacaoyères vieillissantes ont perdu chaque année un peu de leur force de travail familiale, avec tel fils ou neveu quittant l'exploitation cacaoyère du père ou de l'oncle pour aller créer une plantation d'anacardiers, parfois aussi de cacaoyers au village d'origine.

Phénomène encore plus novateur, depuis 3 à 4 ans se forgent de nouveaux flux de travail temporaire. Hommes et surtout femmes quittent le village cacaoyer en février pour aller participer à la collecte des anacardes dans leur village d'origine ou dans un autre village de savane. Ainsi, dans 2 de nos 6 sites d'étude en zone cacaoyère, Bayota et Soubré, ce sont plusieurs bus qui draînent cette force de travail en février vers le nord. Dans deux de nos sites de savane et de contact forêt-savane, Mankono et Konanhiri, les planteurs d'anacardiers comptent sur cette main-d'œuvre pour la récolte par ramassage des noix à terre, travail facile et bien rémunéré par partage de la récolte à parts égales. Certes, les calendriers du cacao et de l'anacarde sont complémentaires. Février-avril est une période creuse pour le cacao. Mais ces flux étaient encore inexistants jusqu'en 2012-2013. Ils ont émergé avec la hausse rapide du prix de l'anacarde de 2014 à début 2018, générant une rémunération plus intéressante que celle des vieilles cacaoyères maintenues à coup de pesticides et d'engrais. Cette inversion des flux reste à quantifier, mais elle contribue à révéler la transition écologique et sociale émergente.

\section{De la mortalité des cacaoyères à l'adoption de l'anacardier en zone forestière. Vers un renouveau cacaoyer?}

\subsection{Bonon et le Parc de la Marahoué}

Le Parc de la Marahoué est passé de 88000 ha de forêt protégée en 1974 à 0 ha en 2015 (Kouakou et al., 2018; Krouba et al., 2018). La région de Bouaflé-Bonon est aussi une des premières zones cacaoyères touchées par la maladie du swollen shoot, décimant des centaines d'hectares de cacao dès les années 1990. L'agriculture de cette région a également subi aussi plusieurs périodes de sécheresse, entraînant la destruction de plantations par des incendies incontrôlés. 


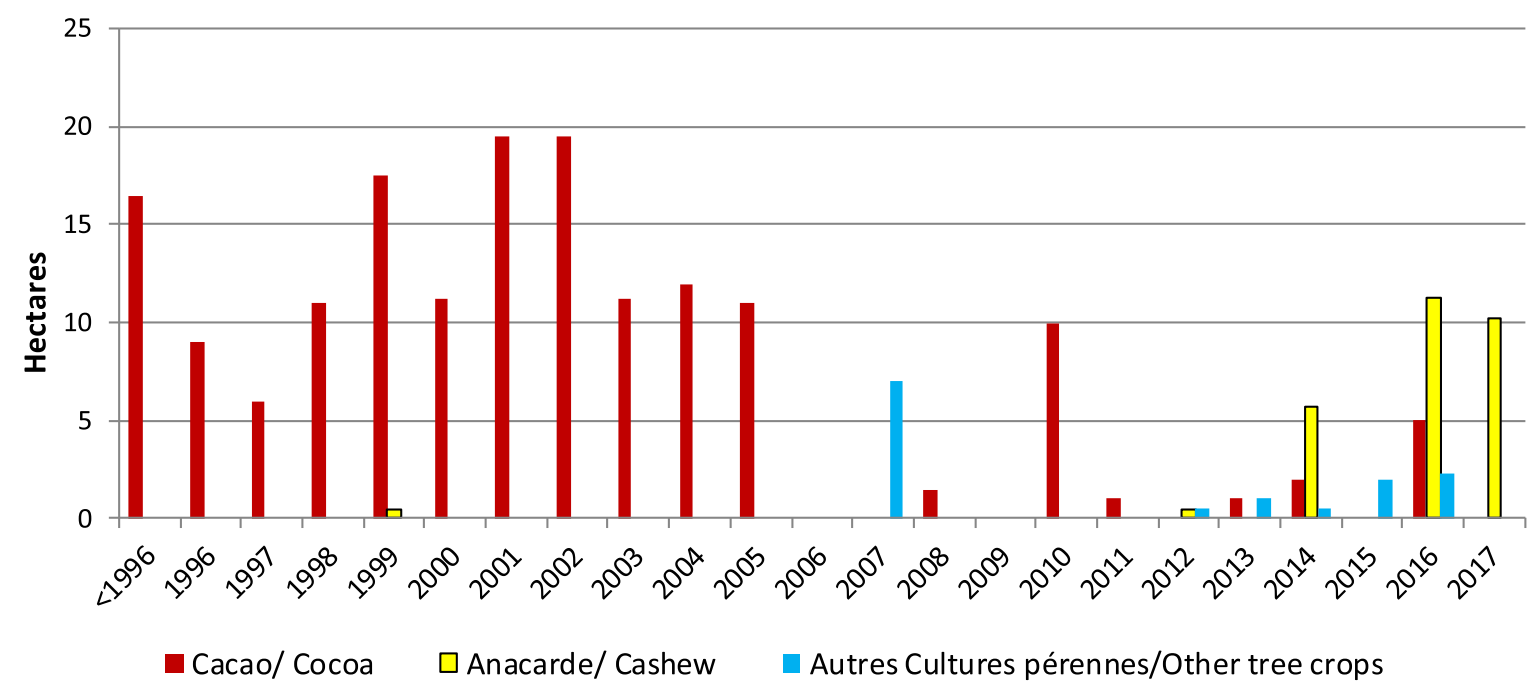

Fig. 6. Surface plantée annuellement et année de début des plantations autour de Bonon (déclarées par les planteurs). Source : Enquêtes auteurs 2017.

Fig. 6. Area planted annually and date of planting around Bonon (declared by cocoa farmers). Source: Authors'surveys 2017.

En réaction à la maladie et à la mortalité des cacaoyers, la première initiative des planteurs immigrants installés a été de défricher des espaces de forêt pour créer de nouvelles cacaoyères qui échapperaient un temps à la dégradation des sols et aux bio-agresseurs. Puis, ils ont été rejoints par de nouveaux migrants chassés par la guerre, déjà planteurs de cacao dans le sud-ouest. Il en est logiquement résulté une déforestation massive du Parc et sa conversion en cacaoyère au cours des années 2000 (Fig. 6). Finalement, au bout de 15 années, le cacaoyer est presque apparu comme une transition passagère entre la forêt (défrichable) et une végétation de jachère faisant suite aux cacaoyères décimées par les maladies et le feu.

Subissant la progression de la mortalité des cacaoyers, la seconde initiative des planteurs a été de diversifier leur assolement en plantant du teck en 2007 et de l'hévéa dans les années 2010. Mais ni l'écologie locale ni le marché n'étaient très favorables à ces cultures.

À peu près au même moment, la troisième initiative des planteurs a été de miser sur l'engrais, en particulier l'engrais foliaire, espérant ainsi reconstituer l'appareil végétatif des cacaoyers. Entre 2009 et 2015, selon nos enquêtes dans la zone de Bonon, $15 \%$ des planteurs ont fait l'effort d'investir dans un engrais foliaire, mais sans grand succès. En 2016, $10 \%$ ont alors essayé les engrais granulés, type 0-23-19. En 2016, seulement $25 \%$ des planteurs interrogés à Bonon déclaraient garder confiance dans cette culture. Soixante-quinze pourcent évoquaient leur découragement face aux sécheresses répétées et à la progression du swollen shoot.

Finalement, l'anacardier apparaît comme la quatrième initiative et innovation majeure qui semble la plus prometteuse. En 2012, un premier innovateur sème des graines d'anacardier dans les «trous de plantation», espaces vides créés par mortalité des cacaoyers. Quelques planteurs l'imitent en 2014 , et finalement, près de $50 \%$ des planteurs interrogés, tous producteurs de cacao à l'origine, tentent à leur tour la plantation d'anacardiers en 2016 et 2017.
Fin 2017, dans $20 \%$ des cas d'adoption de l'anacardier, il semblait s'agir d'une reconversion: une monoculture d'anacardiers remplace l'ancienne monoculture de cacaoyers condamnée par la sécheresse et le swollen shoot. Mais dans $80 \%$ des cas, malgré la déception vis-à-vis du cacao, les planteurs ont l'idée de réintroduire des cacaoyers sous les anacardiers, qui protégent les jeunes plants de cacaoyers de remplacement. Les planteurs pensent que le swollen shoot est associé au vieillissement des cacaoyers et espèrent que les jeunes résisteront à la maladie et à la sécheresse sous l'ombrage des anacardiers. L'agriculture familiale reproduit une de ses stratégies classiques déjà pratiquées dans les années 1970 avec le caféier et le cacaoyer, et plus récemment avec le cacaoyer et l'hévéa: ne sachant quelle culture va réussir, ils associent provisoirement les deux et procèdent à des choix quelques années plus tard.

Dans le «parc» de la Marahoué, tout se passe comme si la rente forêt avait été consommée par le cacaoyer et détruite en moins de 15 ans. Si la validité de ce nouveau système agroforestier combinant anacardiers et cacaoyers se confirme, cette innovation « association anacardier-cacaoyer » représente une transition agroécologique dans tous les sens du terme. L'innovation ne nécessitant pas d'intrants chimiques est essentiellement paysanne, avec peu ou pas d'intervention de la recherche et des structures de conseil, tant publiques que privées.

\subsection{Diversification agroforestière dans les zones historiques de production cacaoyère}

Des processus d'innovation similaires ont été observés plus au sud, au cœur de la zone cacaoyère: l'avancée de la maladie du swollen shoot et la sécheresse de 2016 ont généré le même effet d'adoption rapide de l'anacardier, avec pour l'instant le même double espoir de recréer un environnement agroforestier protégeant le cacaoyer... et le patrimoine terre. 
Tableau 4. Structure des exploitations villageoises à Zatta (Yamoussoukro).

Table 4. Structure of village farms in Zatta (Yamoussoukro).

\begin{tabular}{llcccccc}
\hline & Cacaoyer & Caféier & Hévéa & Palmier & Anacardier & $\begin{array}{c}\text { Total cultures pérennes } \\
\text { autres que cacaoyers }\end{array}$ \\
\hline Superficie moyenne (ha) & 2,32 & 0,37 & 0,01 & 0,01 & 0,24 & 0,63 & 0,11 \\
Écart-type & 1,68 & 0,85 & 0,07 & 0,07 & 0,58 & 1,01 & 0,19 \\
\% de planteurs adoptants & 96 & 28 & 2 & 2 & 25 & 32 \\
\hline
\end{tabular}

Source : enquêtes auteurs 2016-2017 (89 planteurs interrogés).

Tableau 5. Structure des exploitations villageoises à Konankouassikro (Bayota-Gagnoa).

Table 5. Structure of village farms in Konankouassikro (Bayota-Gagnoa).

\begin{tabular}{|c|c|c|c|c|c|c|}
\hline & Cacaoyer & Caféier & Hévéa & Palmier & Anacardier & $\begin{array}{l}\text { Total cultures pérennes } \\
\text { autres que cacaoyers }\end{array}$ \\
\hline Superficie moyenne & 3,74 & 0,0 & 0,33 & 0,0 & 1,31 & 1,64 \\
\hline$\%$ de planteurs adoptants & 100 & & 20 & & 76 & 78 \\
\hline
\end{tabular}

À Zatta, près de Yamoussoukro, l'information sur l'intérêt de l'anacardier vient de manœuvres originaires de la zone de savane. Un premier innovateur, planteur précoce d'anacardiers, s'est lancé en 1999. Sa réussite en 2013-2014, ainsi que la sécheresse de 2015-2016 ayant entrainé une forte mortalité des jeunes cacaoyers, ont généré un effet d'imitation très important. En 2017, $25 \%$ de planteurs avaient déjà adopté l'anacarde (Tab. 4). Dans le village de Konankouassikro, près de Bayota et Gagnoa, au cœur du bassin de production de cacao également touché par les sécheresses, les incendies de plantation et la maladie du swollen shoot, le taux d'adoption de l'anacardier atteint $76 \%$ (Tab. 5). Ce taux place l'anacardier loin devant l'hévéa, lequel paraissait pourtant la principale culture de diversification jusqu'en 2015.

Dans d'autres villages comme dans la région d'Issia, 30\% des anacardiers sont dispersés à densité lâche dans certaines cacaoyères et $70 \%$ sont plantés à forte densité en culture pure à partir d'espace en jachère. Dans cette région, l'incertitude foncière joue. Aucun planteur immigrant n'en parle facilement, mais l'investissement dans la plantation de vieilles parcelles en anacardiers répond aussi à la crainte de ne pas laisser le terrain en jachère trop longtemps, de peur que les autochtones viennent le reprendre pour le revendre à quelqu'un d'autre.

Depuis des décennies, dans plusieurs régions, surtout au sud de la Côte d'Ivoire, l'expansion des cultures pérennes réduit les terres disponibles pour les cultures vivrières et induit une insécurité alimentaire (Koffi et al., 2018). La «transition écologique anacarde » y contribue désormais. Les planteurs en sont bien conscients puisqu'ils introduisent quelques buttes d'igname kokoassie dans les plantations d'anacardiers adultes et que pendant les deux premières années, les jeunes plants d'anacardiers sont associés à des cultures vivrières, même si les terres sont «fatiguées » (igname, bananiers s'ils poussent, gombo, arachide, manioc). Nous retrouvons le même processus, plus avancé, dans le village «PK11» dans la région de Vavoua, en zone de contact forêt-savane. L'association semble fonctionner. Et au moment où nous apportons les dernières corrections à cet article, nous découvrons que les universités de Daloa et de Bouaké sont en train d'observer les mêmes tendances sur toute la région de Vavoua (Koffi, 2019).

\section{Discussion et conclusion}

À partir d'une introduction organisée par les Eaux et Forêts, remontant aux années 1950, le boom récent de l'anacardier en Côte d'Ivoire relève d'une dynamique villageoise, quasi autonome et sans appui conséquent des structures d'appui au monde agricole. Ce boom démontre d'abord la capacité d'innovation et d'entreprise des agriculteurs villageois. Les politiques publiques d'appui à la diversification agricole se sont limitées, dans le cas de la filière anacarde, à l'introduction du matériel végétal dans les années 1950. Alors qu'au Burkina Faso voisin, les structures publiques font partie du système d'innovation «anacarde» (Audouin et Gazull, 2014), elles en sont globalement absentes en Côte d'Ivoire. La seule intervention significative du Conseil $\mathrm{du}$ coton et de l'anacarde en faveur de l'anacarde est une incitation fiscale pour les entrepreneurs de la transformation, en 2019 (au moment où nous apportons les dernières corrections à l'article). Mais du côté des planteurs, il en va tout autrement. La principale intervention de l'État est une lourde taxation, de l'ordre de $100 \mathrm{Fcfa} / \mathrm{kg}$, soit 100 à $200 \%$ du prix touché par le producteur en 2019.

Cet article montre que l'investissement dans la plantation d'anacardiers relève d'innovations paysannes et résulte de la combinaison de trois à quatre facteurs principaux : le marché, la dégradation environnementale structurelle (Boserup, 1965) et les incertitudes foncières. Le quatrième facteur est celui des réseaux familiaux et des flux d'information et de travail entre la zone cotonnière au nord et la zone cacaoyère au sud.

Ainsi, ce boom de l'anacarde s'explique en partie par le marché: envolée des prix de l'anacarde en 1994-2000 et 2014-début 2018, stagnation du prix du coton depuis 2012, hausse du coût des intrants, et donc du coût de production pour 
le coton mais aussi pour le cacao. La première conséquence «agroécologique » du passage du cotonnier ou du cacaoyer à l'anacardier est celle de la disparition du recours aux intrants chimiques. Pour l'instant, l'anacardier se passe d'intrants. C'est une culture dont l'adoption requiert de la terre, un peu de travail mais pas de capital financier lors de l'installation par semis, ce que recherche l'agriculture familiale pour réduire les risques.

La dimension sociale de l'adoption massive de l'anacarde est largement confirmée, via le principe de l'arbre marqueur de terre, aidant le planteur à protéger son patrimoine terreplantation, tant en zone cotonnière qu'en zone cacaoyère. Sur cette fonction d'investissement dans l'arbre pour réduire l'insécurité foncière, l'anacardier remplace ou accompagne le cacaoyer, qui a joué ce rôle pendant des décennies (Chauveau, 2002 ; Colin et al., 2009; Colin, 2018; Ruf, 1995), mais qu'il ne peut plus jouer du fait des mortalités à la replantation postforestière.

Mais pour une large part, en particulier dans le cas des situations de réimplantation du cacaoyer, la diversification par l'adoption de l'anacardier constitue une transition écologique de par sa résistance à la sécheresse, par l'absence de maladies ou d'insectes nuisibles majeurs, et par sa fonction de protection du jeune cacaoyer. D'une certaine façon, avec l'effondrement des cours de la noix de cajou, le cycle de l'anacardier revient en partie vers sa fonction initiale de fournisseurs de services écologiques. De ce point de vue, notre étude débouche sur deux hypothèses à explorer par rapport au changement climatique. D'une part, le swollen shoot, à l'incidence clairement accélérée par les sécheresses, servirait d'aiguillon à des stratégies paysannes visant à limiter l'impact du changement climatique : l'anacardier deviendrait un outil majeur de cette stratégie. D'autre part, en symétrie à la baisse des précipitations et à la déforestation dans le sud, y aurait-il un lien entre la «reforestation» massive des régions de savane par l'anacardier et la légère augmentation des précipitations qui y serait observée actuellement?

Finalement, le boom de l'anacarde illustre la thèse défendue: l'adoption d'une nouvelle culture pérenne représente une transition agraire face à des contraintes climatiques, écologiques et sociales, une transition probablement provisoire, en permettant de ré-explorer et ré-exploiter une part de ressources naturelles qui ne sont plus à la portée de la culture précédente (le cacaoyer ou le cotonnier). C'est déjà le cas des plantations d'anacardiers en culture «quasi pure» (mais avec déjà un potentiel agroforestier quand des ignames kokoassie s'y insèrent), contribuant à convertir les paysages de savanes en paysages forestiers.

De surcroît, l'association entre anacardiers et cacaoyers pourrait offrir une seconde chance à la cacaoculture dans les zones où sa replantation échouait auparavant: une innovation paysanne par excellence. Elle est aussi agroforestière. Certes, personne ne sait encore si les planteurs garderont les anacardiers une fois les cacaoyers consolidés dans leur croissance, mais cette forme de rotation et relais de culture dans le temps relève précisément d'une logique agroforestière, la «transition anacardière ».

L'industrie du chocolat promet de se lancer dans une cacaoculture durable et sans déforestation. Avec l'association anacardier-jeune cacaoyer, l'agriculture familiale n'est-elle pas en train de l'inventer avec quelques années d'avance sur les structures publiques et privées? Néanmoins, en zone forestière comme en zone de savane ivoiriennes, prenons garde à l'insécurité alimentaire que pourrait engendrer le développement de systèmes affichés «sans déforestation» et labellisés durables, à l'intention des consommateurs des pays importateurs de ces produits. C'est le danger structurel des cycles de cultures pérennes quand leurs cours s'effondrent. Les tensions en termes de sécurité alimentaire, vitale pour la résilience de l'agriculture familiale, pourraient s'accroître notablement si l'anacardier concernait trop de surface cultivable, au sud et surtout au nord de la Côte d'Ivoire.

Remerciements. La lecture attentive de deux référés anonymes et celle des éditeurs de ce numéro thématique, Ludovic Temple et Hubert de Bon, a permis d'améliorer très significativement l'article et nous les en remercions vivement. Je remercie également Michel Fok pour avoir partagé ses données de prix sur le coton, ainsi que Pierre Ricau et Nitidae (ex. Rongead) pour leur communication de données de prix et échanges sur la filière anacarde à partir de leurs remarquables bulletins de veille sur la noix de cajou en Afrique de l'Ouest.;

\section{Références}

Audouin S, Gazull L. 2014. Les dynamiques d'un système d'innovation à travers le prisme des diffusions spatiales. Le cas de l'anacarde au Sud-Ouest du Burkina Faso. L'Espace géographique 43(1): 35-50.

Banque Mondiale. 2015. Situation économique en Côte d'Ivoire. Banque Mondiale, Abidjan, Côte d'Ivoire.

Basset TJ. 2009. Mobile pastoralism on the brink of land privatization in Northern Côte d'Ivoire. Geoforum 40(5): 756-766. http://www. sciencedirect.com/science/article/pii/S0016718509000438.

Boserup E. 1965. The conditions of agricultural growth: the economics of agrarian change under population pressure. Londres, Grande-Bretagne: Allen et Unwin.

Chauveau JP. 2002. Question foncière et construction nationale en Côte d'Ivoire. Politique Africaine 78: 94-125.

Colin JP, Le Meur PY, Léonard E. 2009. Les politiques d'enregistrement des droits fonciers : du cadre légal aux pratiques locales. Hommes et Sociétés. Paris, France: Karthala.

Colin JP. 2018. Investissements dans les agricultures familiales d'Afrique subsaharienne, sécurité foncière et formalisation des droits. Comité technique «Foncier et Développement», AFD, 4 p. www.foncier-developpement.fr.

Cretenet M, Vaissayre M. 1986. Modèle de décision appliqué à l'interaction entre fertilisation minérale et protection phytosanitaire en culture cotonnière. Cot. Fib. Trop. XLI(2): 89-96.

Diomandé M. 2002. La culture de l'anacarde dans le développement de la région de Bondoukou. Communication à la conférence internationale sur l'avenir des cultures pérennes. Yamoussoukro, 5-9 nov 2001. CIAD/ESI-VI, BNETD, 16 p. ISBN: 2-87614$502-2$.

Dugué P. 2002. Dynamiques de plantation et durabilité des systèmes de cultures pérennes en zone de savane de Côte d'Ivoire. Communication à la conférence internationale sur l'avenir des cultures pérennes. Yamoussoukro, 5-9 nov 2001. CIAD/ESI-VI, BNETD, $14 \mathrm{p}$.

ENGREF. 2002. Parc national de la Marahoue. Étude préalable à un aménagement du parc et de sa zone périphérique. Montpellier, France: ENGREF, 114 p. 
Fiko K, Yao A. 2013. L'hévéa dans le temple du cacao. Le sud-ouest de la Côte d'Ivoire au cours de la période 1990-2000. In : Ruf F, Schroth G, eds. Les cultures pérennes tropicales. Enjeux économiques et écologiques de la diversification. Versailles, France: Quae, pp. 119-130.

Fok M, Kone S, Akindes F, Soro M. 2015. Mission d'observation des exploitations cotonnières en Côte d'Ivoire. Abidjan, Côte d'Ivoire: Technoserve.

Kone S. 2014. Diagnostic des systèmes de production à base d'anacardiers. Yamoussoukro, Côte d'Ivoire: ESA et FIRCA.

Koffi GRY, Kouassi K, Barima YSS, Kpangui KB, Assi-Kaudjhis JP. 2018. Pratique cacaoyère, délitement de l'accès au foncier et sécurité alimentaire dans la sous-préfecture de Dania. Tropicultura 36: 380-391.

Koffi GRY. 2019. Économie de plantation et sécurité alimentaire dans la sous-préfecture de Dania (centre-ouest de la Côte d'Ivoire). Thèse de doctorat, Mention Géographie humaine. Bouaké, Côte d'Ivoire: Université Allassane Ouattara, 479 p.

Kouakou ACA, Coulibaly B, Kaba D, Anoh KP, Courtin F. 2018. Dynamique de peuplement et modification paysagère dans le parc national de la Marahoué (Côte d'Ivoire). In : Barima, Bamba, eds. Conflits, dynamiques des paysages et sécurité alimentaire. Tropicultura 36: 206-216.

Krouba GID, Ouattara AA, Kouakou ACA, Adopo ARI, Fauret P, Coulibaly B, et al. 2018. Dynamiques de peuplement et modifications paysagères dans la zone rurale sud de la ville de Bonon en 2000 et 2015. Tropicultura 36: 271-280.

Léonard E, Oswald M. 1996. Une agriculture forestière sans forêt. Changements agro-écologiques et innovations paysannes en Côte d'Ivoire. Nature, sciences et sociétés 4(3): 202-216.

Losch B. 1990. La filière caoutchouc naturel en Côte d'Ivoire: originalité et enjeux. In : Griffon M, ed. Economie des filières en régions chaudes: formation des prix et échanges agricoles. Montpellier, France: CIRAD-MESRU, p. 785-804. http://agri trop.cirad.fr/429724/1/document_429724.pdf.

Ouattara GM, Coulibaly PJM, Seri K, Soro HZ, Coulibaly H, Touré LF, et al. 2012. Guide pratique de l'anacarde. Entretien des parcelles. Abidjan, Côte d'Ivoire: GIZ \& OLAM, 6 p.

Oura Kouadio R. 2017. Conflits fonciers autour de l'activité hévéicole dans la sous-préfecture de Daoukro (centre-est Côte d'Ivoire). Baobab 21: 151-162.

RufF. 1995. Boums et crises du cacao. Les vertiges de l'or brun. Paris, France: Karthala.

RufF. 1999. Comment et pourquoi la Côte d'Ivoire produit durablement plus d'un million de tonnes de cacao? Afrique Agriculture 268: 21-25. https://www.researchgate.net/publication/299456711.

Ruf F. 2012. L'adoption de l'hévéa en Côte d'Ivoire. Prix, mimétisme, changement écologique et social. Economie Rurale 330-331: $103-$ 123. https://journals.openedition.org/economierurale/3527.

Ruf F. 2016. Une femme en Côte d'Ivoire, une femme au Burkina Faso. Changement écologique et social autour du cacao... et de l'anacarde. EchoGéo [En ligne], 37 | 2016, mis en ligne le 06 octobre 2016. http://echogeo.revues.org/14696.

Ruf F. 2018. Crises politico-militaires et climatiques en Côte d'Ivoire, 2000-2017. Du cacao à l'anacarde, de la rente forêt à la fumure animale. In : Barima, Bamba, eds. Conflits, dynamiques des paysages et sécurité alimentaire. Tropicultura 36: 281-298.

Ruf F, Schroth G. 2013. Les cultures pérennes tropicales. Enjeux économiques et écologiques de la diversification. Versailles, France: Quae.

Sanial E. 2018. L'appropriation de l'arbre, un nouveau front pour la cacaoculture ivoirienne ? Contraintes techniques, environnementales et foncières. Cahiers Agricultures 27(5): 55005. DOI: 10.1051 /cagri/2018036.

Citation de l'article : Ruf F, Kone S, Bebo B. 2019. Le boom de l'anacarde en Côte d'Ivoire : transition écologique et sociale des systèmes à base de coton et de cacao. Cah. Agric. 28: 21. 